\title{
Spatio-temporal pattern of viral meningitis in Michigan, 1993-2001
}

\author{
Sharon K. Greene ${ }^{1}$, Mark A. Schmidt ${ }^{1,2}$, Mary Grace Stobierski ${ }^{2}$, \\ and Mark L. Wilson ${ }^{1}$ \\ ${ }^{1}$ Department of Epidemiology, University of Michigan School of Public Health, Ann Arbor, MI \\ 48109, USA (e-mail: skgreene@umich.edu) \\ ${ }^{2}$ Division of Communicable Disease and Immunization, Michigan Department of Community \\ Health, Lansing, MI 48913, USA
}

Received: 13 November 2003 / Accepted: 25 October 2004

\begin{abstract}
To characterize Michigan's high viral meningitis incidence rates, 8,803 cases from 1993-2001 were analyzed for standard epidemiological indices, geographic distribution, and spatio-temporal clusters. Blacks and infants were found to be high-risk groups. Annual seasonality and interannual variability in epidemic magnitude were apparent. Cases were concentrated in southern Michigan, and cumulative incidence was correlated with population density at the county level $(r=0.45, p<0.001)$. Kulldorff's Scan test identified the occurrence of spatio-temporal clusters in Lower Michigan during July-October 1998 and $2001(p=0.01)$. More extensive data on cases, laboratory isolates, sociodemographics, and environmental exposures should improve detection and enhance the effectiveness of a SpaceTime Information System aimed at prevention.
\end{abstract}

Key words: Communicable disease, Epidemiology, Space-time clustering, Spatial analysis, Viral meningitis

JEL Classification: I10, I19

\section{Introduction}

Viral meningitis, also known as aseptic or nonpurulent meningitis, is an infection of the meninges that may result in severe systemic disease and

The authors gratefully acknowledge Dr. Duane Newton, former manager of the Virology Section, Michigan Department of Community Health Bureau of Laboratories, for his provision of data on viral isolates. We also appreciate the contributions of Drs. Leah Estberg and Dunrie Greiling for ClusterSeer support, Scott Swan for his assistance with ArcView GIS, and Dr. Edward L. Ionides for his advice on time series analysis. We are grateful to Dr. Pierre Goovaerts and two anonymous reviewers for their insightful comments on an earlier draft of this paper. This project was supported in part by a grant from the National Oceanic and Atmospheric Administration's Joint Program on Climate Variability and Human Health, a consortium including the EPA, NASA, NSF, and EPRI (NA16GP2361) to MLW. 
neurological damage, particularly in the very young (Rotbart 2000, ProMED-mail 2003). Viral meningitis has been linked with an estimated 26,000-42,000 hospitalizations nationally each year (CDC 2003). Enteroviruses are the most common cause of viral meningitis in the U.S., accounting for $85-95 \%$ of all cases with an identified cause (Rotbart 2000). A total of 64 enterovirus serotypes are recognized, including 61 non-polio enteroviruses (CDC 2002). Of these, echoviruses (types 2, 5, 6, 7, 9, 10, 11, 14, 18, and 30) cause about half of cases, and coxsackieviruses (group B, types 1-6) are responsible for another third of cases (Chin 2000). Sporadic disease may be due to coxsackievirus group A, arboviruses, measles virus, herpes simplex virus, varicella virus, lymphocytic choriomeningitis virus, adenovirus, and others (Chin 2000). Outbreaks can be caused by any one of these viruses or a combination thereof, and laboratory typing is seldom routine, so the causes behind periods of elevated incidence are often left unexplained. Infection is common although clinical illness is rare, as fewer than one in 500 enterovirus infections actually results in viral meningitis (CDC 2000a).

Enteroviruses causing viral meningitis are transmitted via various routes, including fecal-oral, water, food, air, inoculation, and blood. Fecal-oral transmission is the classic means of enterovirus spread, and is common wherever hygiene and sanitation are inadequate, as is sometimes the case among children in day-care centers. Air-borne transmission may predominate in other settings (Morens and Pallansch 1995). Common symptoms include malaise, fever, headache, stiff neck, abdominal pain, nausea, and vomiting. Patients may also develop sore throat, chest pain, photophobia, or a maculopapular rash (Huether and McCance 2000). Cerebrospinal fluid of patients with viral meningitis is characterized by the absence of bacteria that cause these symptoms, and typically the presence of signs such as pleocytosis, increased protein, and normal sugar. Active illness usually lasts $<10$ days, and recovery is often complete within a few weeks, although irritability and fatigue may persist (Chin 2000). Treatment is symptomatic (CDC 2003). Five to ten percent of patients experience complications including febrile disorders, movement disorders, lethargy, complex seizures, or coma (Modlin 2004).

Viral meningitis cases are highly seasonal, although the mechanisms underlying this observation are unknown. In temperate climates, enteroviral disease incidence generally increases during late summer and early autumn (Chin 2000). This is potentially because fecal-oral transmission of enteroviruses is aided by warm weather and sparse clothing, especially among children (Rotbart 2000). The predominant serotypes of enteroviruses circulating in a community during a given year cycle with varying periodicity. Outbreaks coincide with the availability of new susceptible hosts (e.g. children not previously exposed to a particular virus). Serotypes responsible for elevated incidence frequently cause disease in the young. A serotype that was absent from a community for several years and reintroduced will affect older children and adults who have never been exposed (Rotbart 2000). Thus, analyses of the temporal pattern of incidence should shed light on risk factors for symptomatic disease.

Young age and immunodeficiency are considered risk factors for viral meningitis. For unknown reasons, higher infection rates appear to occur 


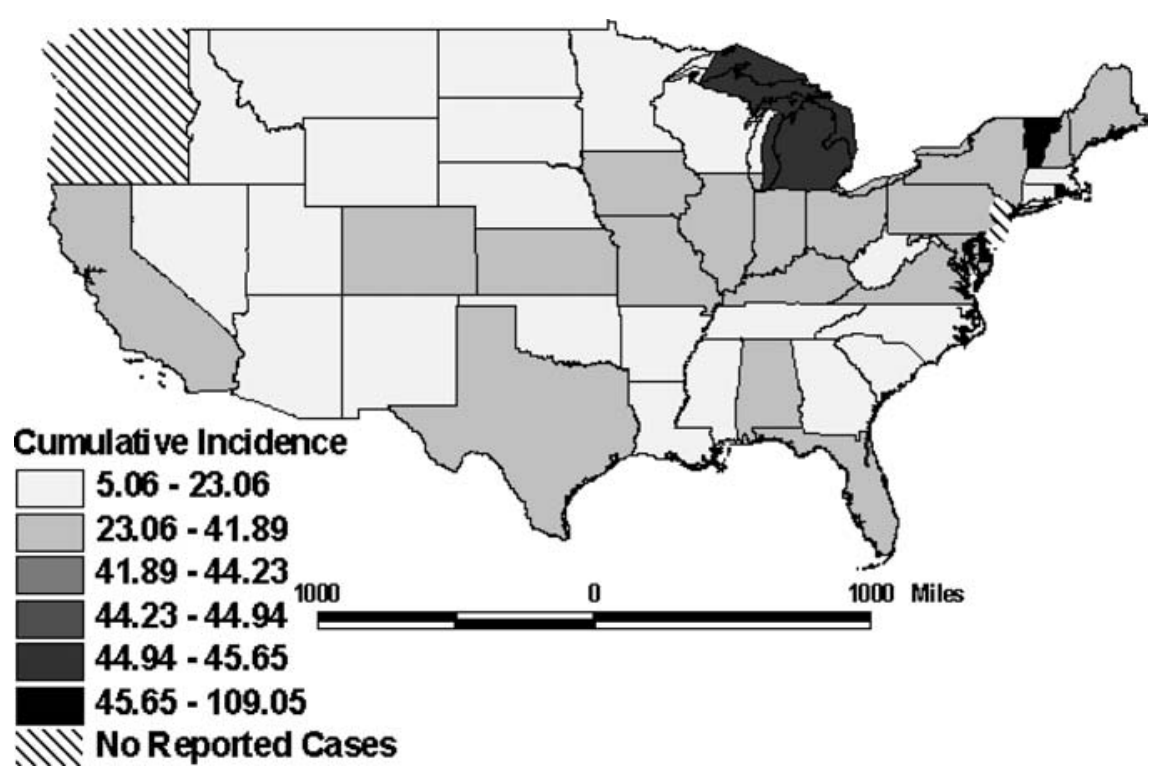

Fig. 1. Cumulative incidence per 100,000 of viral meningitis by state, continental United States, 1989-1994 (CDC 1995)

among males, as well as among those who are of lower socioeconomic status and live in crowded areas, due to the increased potential for fecal contamination (Modlin 2004; Morens and Pallansch 1995).

Viral meningitis ceased to be a nationally notifiable disease in 1995 (CDC 2001), thereby limiting investigations into its national distribution. United States case data through 1994 (CDC 1995) indicate that viral meningitis is present throughout the country, but the incidence in Michigan historically far exceeds that of most of the nation (Fig. 1). Fortunately, the Michigan Department of Community Health (MDCH) continues to collect data on viral meningitis cases.

Accordingly, we undertook a spatio-temporal analysis of the patterns of viral meningitis cases in Michigan from 1993 to 2001. Various investigations have applied spatial analytic techniques to other diseases at the county-level in efforts to improve understanding of transmission dynamics in a state. For example, methods such as the Ederer-Myers-Mantel and Moran's I procedures have been applied to human brucellosis in California, identifying Hispanics as the subpopulation most at risk for infection (Fosgate et al. 2002). In addition, the spatial autocorrelation of Lyme disease has been investigated in New York state at the county scale, helping to determine a characteristic spatial scale for infection patterns, to quantify the extent and intensity of clustering about disease foci, and to suggest a scale for control efforts (Glavanakov et al. 2001). In this study, we evaluated viral meningitis incidence throughout Michigan using similar tools and analytic methods in order to explore incidence patterns and illustrate applications of spatiotemporal techniques. Any disease that is prone to outbreaks can be better understood by clarifying disease occurrence in terms of person, place, and time and exploring spatio-temporal patterns. Areas identified as having high 
incidence may be further targeted for heightened surveillance, education, and prevention efforts. Specifically, viral meningitis cases from 1993 to 2001 were analyzed using standard epidemiological methods, mapped with a geographic information system (GIS), and then further analyzed using spatial and temporal cluster statistics. These approaches, if applied to ongoing data collection, could help lay the foundation for a Space-Time Information System (STIS) that jointly considers the spatial and temporal patterns of infectious disease, aids in the determination of etiology and risk factors, guides outbreak investigations, and, ultimately, may reduce disease incidence.

\section{Materials and methods}

\subsection{Study site and population}

Roughly 10 million people lived in Michigan in the year 2000. The state's 56,804 square miles are divided by the Great Lakes into two main landmasses: the sparsely populated Upper Peninsula (UP) has a humid climate with long severe winters and short mild summers, while Lower Michigan has more moderate weather, with higher summer temperatures, less severe winter temperatures, and less snowfall. Generally, the Great Lakes serve to moderate cold temperatures (UniversalMAP 2000). Michigan's population is most dense in the southeast; $9.6 \%$ of the state's residents live in Detroit (U.S. Census). For this study, age-specific population counts for each Michigan county were drawn from the 1990 U.S. Census, and total counts for each county were obtained from the 2000 U.S. Census. Annual viral meningitis incidence in children $<10$ years old ( $n=3,031$ cases) was compared using 1990 vs. intercensal county-specific population denominators, and the incidences were highly correlated $\left(\mathrm{r}^{2}=0.99\right.$, $\mathrm{p}$-value $\left.<0.0001\right)$. Based on these data, it was determined that simply age-adjusting incidence rates to the 1990 Census population was sufficient.

\subsection{Surveillance data}

The MDCH routinely collects data on all diagnoses of reportable diseases or conditions, as defined by the Michigan Public Health Code (Michigan Compiled Laws 1978). The MDCH provided information on 8,803 viral meningitis cases reported though the state's passive disease surveillance system from 1993 to 2001. A confirmed viral meningitis case was defined as "a clinically compatible case diagnosed by a physician as aseptic meningitis, with no laboratory evidence of bacterial or fungal meningitis" (CDC 1997). This is essentially a diagnosis of exclusion. The only city in Michigan that reported cases independently from its county was Detroit, which is within Wayne County. Eleven of 83 counties did not report any cases over the study period, seven of which are in the UP and four in Lower Michigan. Reporting was nearly complete for most case characteristics, including date of report $(100 \%)$, county of residence $(100 \%)$, age $(99.9 \%)$, and sex $(99.2 \%)$, but less so for race $(69.4 \%)$. 
Prior to the time frame of this study, total annual counts of viral meningitis in Michigan with no individual-level case information were available from MDCH (1984-1992). To enable a comparison of incidence with nearby states, annual counts were obtained for Illinois from 1990 2000 (Illinois Department of Public Health website: http://www.idph.state.il.us/health/infect/communicabledisease.htm) and for Indiana from 1990 2001 (personal communication via electronic mail, Julia Butwin, March 2002).

Virus type identification was obtained through the MDCH Bureau of Laboratories for isolates of some viral meningitis cases during JuneDecember 2001. Samples or specimens from 114 such patients were obtained from cerebrospinal fluid (most commonly), bronchial fluid, throat, stool, or vagina. Antibodies against enteroviral proteins were used as presumptive evidence of the presence of enteroviruses in the isolates. Typing was performed using monoclonal fluorescent antibody testing and pooled antisera. Isolates not typeable by these methods were sent to the Centers for Disease Control and Prevention (CDC) for PCR sequencing. An enterovirus subtype was identified from $43(37.7 \%)$ of these samples.

\subsection{Statistical analyses}

Cumulative incidences and relative risks were calculated using SAS for Windows v8 (SAS Institute, Cary, NC). Disease incidence was mapped using ArcView GIS v3.2a (Environmental Systems Research Institute, Inc., Redlands, CA). Time series analysis techniques were applied to the data using R v1.7.1 (R Foundation for Statistical Computing, Vienna, Austria). Kulldorff's Scan test (Kulldorff 1997) was applied to monthly, county-level incidence using ClusterSeer (TerraSeer Inc., Ann Arbor, MI) to search for spatio-temporal clusters. All non-reporting counties were excluded from the spatio-temporal analysis, as were the counties in the UP because Kulldorff's Scan test could not analyze the space across the water of the Great Lakes within which no one was resident. A total of 8,743 cases remained for inclusion in the spatio-temporal analysis.

\section{Results}

\subsection{Demographics of cases}

A total of 8,803 viral meningitis cases were reported during the 9 years of study. Overall, 4,402 (50.4\%) cases were female and 4,329 (49.6\%) were male, proportions that are representative of the population of Michigan in 2000 (U.S. Census). Cases ranged in age from $<1$ to 93 years (mean $=21.8$ years, $\mathrm{SD}=31.3$ years). The median age of cases (18.0 years) was less than that of the Michigan population in 2000 (35.5 years, U.S. Census). Indeed, 1,345 cases $(15.2 \%)$ involved infants $<1$ year old, representing a cumulative 9-year incidence of 1,040.6 per 100,000. Cumulative incidence for the rest of the population was 81.4 per 100,000 (relative risk [RR] for $<1$ year old $=12.9 ; 95 \%$ confidence interval $[\mathrm{CI}]$ 12.2-13.7). 
Blacks represented 14.2\% of Michigan's population in 2000 (U.S. Census), yet they were disproportionately represented among viral meningitis cases, comprising $2,044(33.5 \%)$ of cases. The cumulative incidence of viral meningitis among Whites was 51.1 per 100,000 , but for Blacks it was 158.2 per 100,000 (RR for Blacks $=3.1 ; 95 \%$ CI 2.9-3.3).

\subsection{Temporal trends}

Cases were seasonally distributed, with more than two-thirds reported during July through October. The cumulative statewide incidence of viral meningitis during these four months, summed over the nine study years and calculated using county-specific 2000 Census population denominators, was 60.2 cases
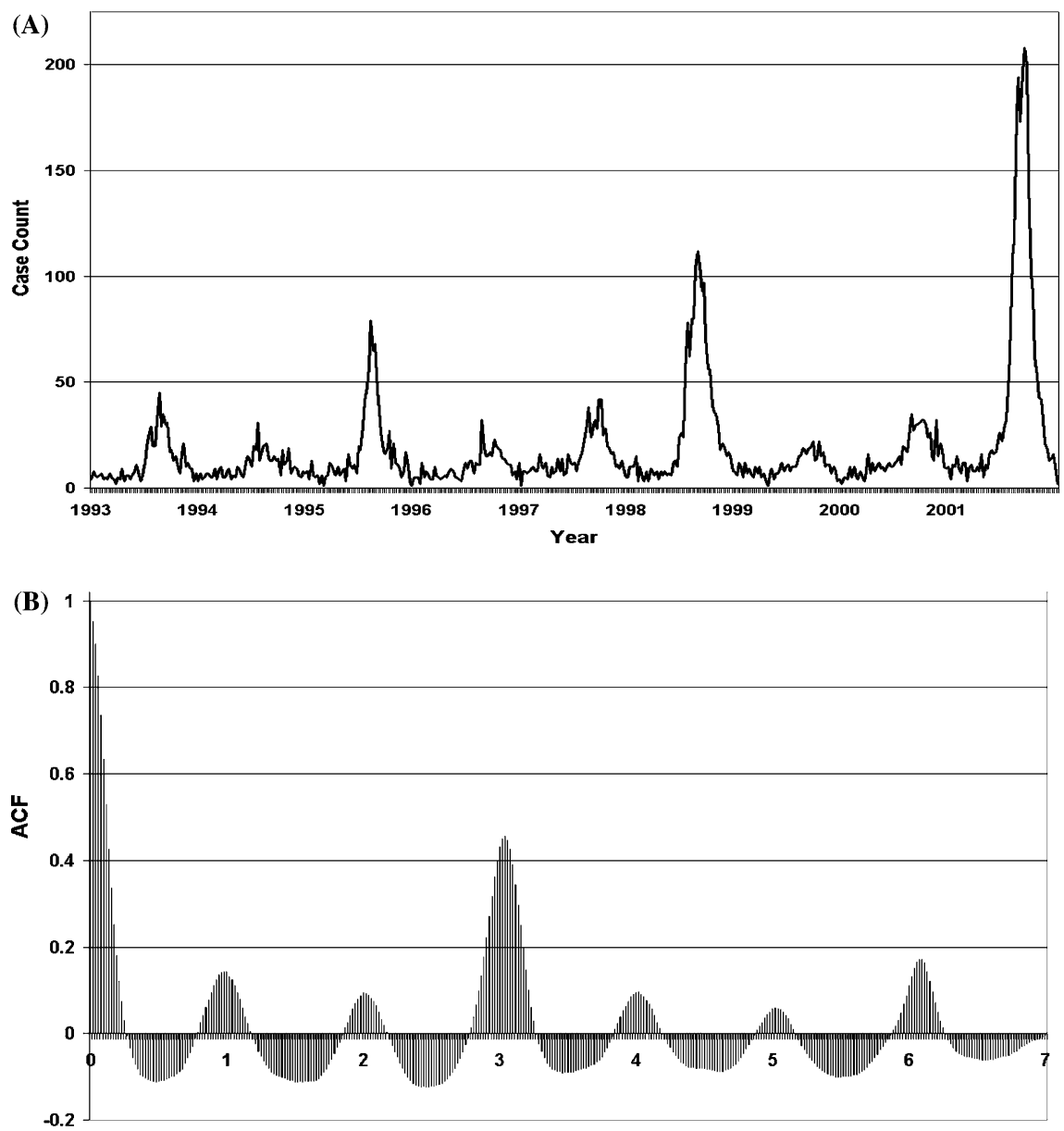

\section{Lag (Years)}

Fig. 2. (A) Weekly number of viral meningitis cases in Michigan, 1993-2001. (B) The autocorrelation function (ACF) from a zero to a seven-year lag 
per 100,000, compared with 28.4 cases per 100,000 from November through June (RR of July-October $=2.1 ; 95 \% \mathrm{CI}=2.0-2.2$ ). Despite strong annual seasonality, considerable inter-annual variability in epidemic magnitude was apparent (Fig. 2A).

Periodic extremes in reported cases of viral meningitis occurred roughly every three years for this time series. The largest epidemics were in 1995, 1998, and (most strikingly) 2001. The autocorrelation function (ACF) revealed a strong autocorrelation of 0.43 at 3 years and 0.16 at its multiple of 6 years (Fig. 2B). However, peak epidemic years in Michigan prior to the beginning of this study in 1993 were 1987 and 1990, suggesting that a regular 3-year cycle may vary somewhat (data not shown). The ACF pattern did not change appreciably when applied to high-risk subsets of the case population such as children $<10$ years-olds and Blacks (results not shown).

\subsection{Spatial trends}

Age-adjusted cumulative incidence tended to be highest in southern Michigan, particularly in the southeast, near and around Detroit (154.9 cases per 100,000; Fig. 3). Other areas of notably high age-adjusted cumulative incidence relative to the surrounding area include the counties of Muskegon (211.8 cases per 100,000) and Newaygo (121.8 cases per $100,000)$ in the western central part of Lower Michigan, Kalamazoo in the

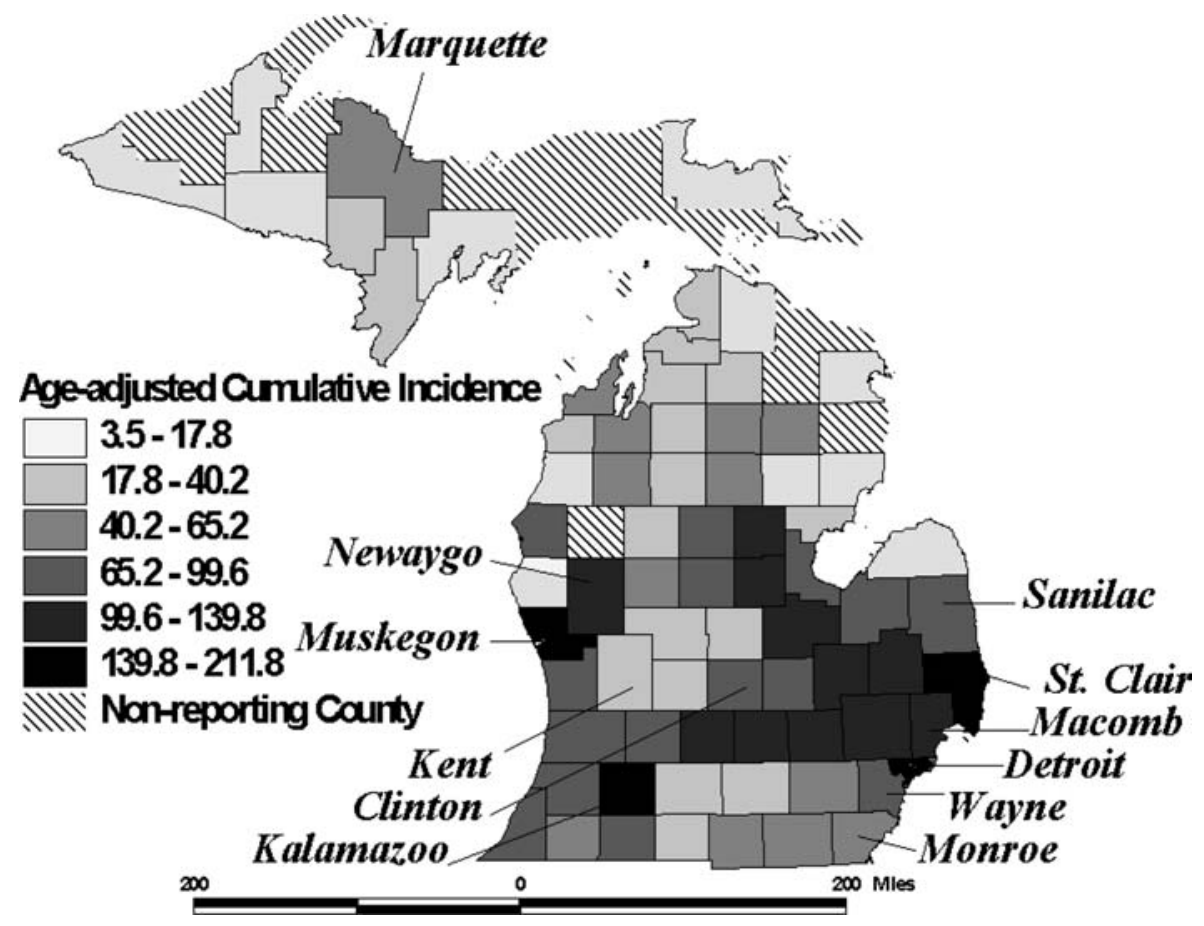

Fig. 3. Cumulative incidence per 100,000 of viral meningitis in Michigan by county, 1993-2001, age-adjusted to the 1990 population. Counties mentioned in text are labeled 
southwest (185.8 cases per 100,000), and Marquette in the UP (57.3 cases per 100,000).

County-specific cumulative incidence (Fig. 3) was similar to the spatial pattern of county-level population density (i.e. number of residents / sq. mi.; results not shown). The Pearson correlation coefficient for cumulative incidence and population density was positive and significant $(\mathrm{r}=0.45$, $\mathrm{p}$-value $=0.0003$ ). This suggests that counties with greater human density tended to have more transmission.

To determine whether there was a spatial pattern in inter-annual variability of incidence, the coefficient of variation (C.V. = standard deviation/mean) for nine years of annual incidence in each county was mapped (Fig. 4), with the understanding that counties with low incidence may yield unstable C.V. estimates. Interestingly, Figs 3 and 4 show essentially opposite patterns, such that the areas of highest incidence (generally Lower Michigan) were less variable, whereas the areas of lower incidence (northern Lower Michigan and the UP) showed greater year-toyear variability.

\subsection{Spatio-temporal trends}

Kulldorff's Scan test identified the three most likely spatio-temporal clusters in the 65 reporting sites of Lower Michigan (Fig. 5). The first most likely

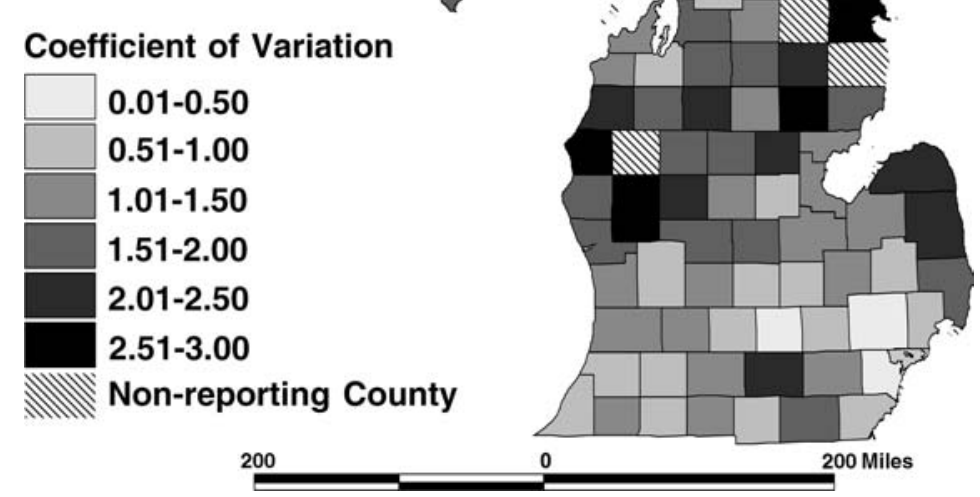

Fig. 4. Coefficient of variation (C.V.) of annual incidence of viral meningitis cases in Michigan by county, 1993-2001. The coefficient of variation for each county was calculated by dividing the standard deviation of that county's annual incidence by its mean annual incidence 


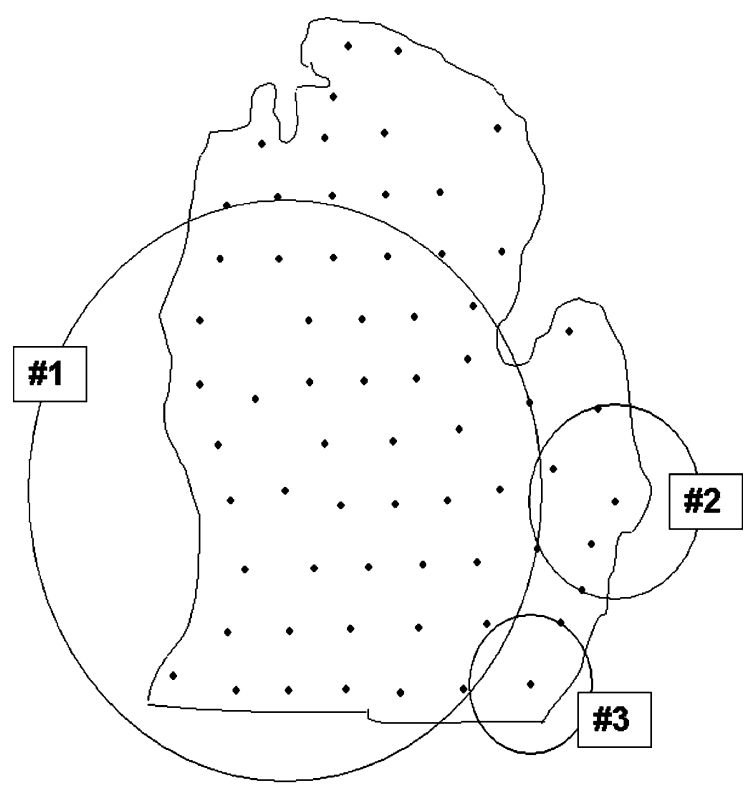

Fig. 5. Spatio-temporal clusters of viral meningitis cases by county in Lower Michigan, 19932001. The three most-likely overall clusters as determined by Kulldorff's Scan test include [\#1] a 42-county region during July to October 2001, [\#2] a five-county region and Detroit from July to October 1998, and [\#3] a two-county cluster from August to October 2001

cluster was a 42-county region centered on Kent County during four months in late summer/early fall 2001. The second most likely cluster was located around St. Clair County during the same period in 1998, and the third most likely was centered on Monroe County in that period in 2001. Each spacetime cluster was significant at $p$-value $=0.01$. This test was also run separately for Blacks and for children aged $<10$ years old. For Blacks, there were only two significant spatio-temporal clusters ( $p$-value $=0.01$ for both): a 37-county region centered on Clinton County during July-September 2001, and solely St. Clair County from June-September 1998.

The most likely cluster for $<10$ year-olds was the same as that found for all cases (42-county region centered on Kent County), but with a slight temporal shift to July-September $2001(\mathrm{p}$-value $=0.01)$. The second and third most likely clusters encompassed 5 counties plus Detroit centered on Macomb County during July-September 1998 (p-value =0.01) and solely Sanilac County during July-September 1998 (p-value $=0.03)$.

Within Cluster \#1 (Fig. 5), children < 10 years old comprised $533(42 \%)$ of 1,268 cases. Of all 8,743 cases included in the spatio-temporal analysis, 3,014 $(34 \%)$ cases were in this age group. Thus, children $<10$ years old were at significantly greater risk for viral meningitis in the first most likely spatiotemporal cluster than they were over the entire region and study period $\left(X^{2}\right.$ for specified proportions $=36.5$, $\mathrm{df}=1$, p-value $<0.0001)$. This information could be beneficial in determining the reasons behind the increased number of cases in this cluster. 
Annual incidences of viral meningitis in Michigan and in Illinois were strongly correlated from 1990-2000 $(\mathrm{r}=0.88$, $\mathrm{p}$-value $=0.0004)$. Both states experienced maximum incidence over this period in 1998 (yet incidence in Michigan was nearly two times greater than that in Illinois). Curiously, the correlation between annual incidences of Michigan and the southern border state of Indiana was non-significant $(\mathrm{r}=0.49, \mathrm{p}$-value $=0.11)$.

\subsection{Laboratory results}

Of the 43 subtyped enteroviral isolates obtained from viral meningitis patients in June-December 2001, 28 (65.1\%) were from children < 10 years old. There were differences between this age group and cases $\geq 10$ years old in the proportions of viral subtypes isolated (Table 1). Although not representative of all viral meningitis infections, these data suggest that younger cases were more likely to be infected with coxsackie B-3 or echovirus 13 , while only older cases harbored echovirus 30. This apparent pattern might be explained by true differences in enterovirus activity, age-specific clinical syndromes of specific enteroviruses, or differences in ease of isolation (Strikas et al. 1986).

\section{Discussion}

To our knowledge, this paper represents the first study that examines spatiotemporal patterns of viral meningitis cases at a resolution as fine as countylevel. Our results confirm the existence of certain high-risk groups and disease clustering in both space and time within Michigan.

Several findings from previous studies of viral meningitis are supported, including risk factors related to seasonality, age, race, and crowding. Temporally, our results are consistent with the seasonal pattern of viral meningitis typically reported from temperate climates, i.e. a peak in the summer and autumn (Rotbart 2000, CDC 2000a, CDC 2001). In a study of enteroviral isolates from across the U.S. collected through the Enterovirus

Table 1. Relative presence of identified causative viruses associated with viral meningitis cases in Michigan, June to December 2001

\begin{tabular}{lll}
\hline Virus & \multicolumn{2}{c}{ Number of Isolates $(\%)$} \\
\cline { 2 - 3 } & Cases $<10$ Years & Cases $\geqslant 10$ Years \\
\hline Coxsackie B-2 & $3(10.7 \%)$ & $1(6.7 \%)$ \\
Coxsackie B-3 & $8(28.6 \%)$ & $1(6.7 \%)$ \\
Coxsackie B-4 & $0(0.0 \%)$ & $1(6.7 \%)$ \\
Echovirus 4 & $3(10.7 \%)$ & $1(6.7 \%)$ \\
Echovirus 6 & $1(3.6 \%)$ & $1(6.7 \%)$ \\
Echovirus 9 & $0(0.0 \%)$ & $1(6.7 \%)$ \\
Echovirus 11 & $4(14.3 \%)$ & $3(6.7 \%)$ \\
Echovirus 13 & $9(32.1 \%)$ & $5(33.3 \%)$ \\
Echovirus 30 & $0(0.0 \%)$ & $15(100.0 \%)$ \\
Total & $28(100.0 \%)$ & \\
\hline
\end{tabular}


Surveillance Program during 1970-1979, 82\% of all enteroviral isolates were submitted from June to October, and the average number of isolates for each of these months was 6.6 times higher than the monthly average for the other seven months (Moore 1982). Generally, viruses carried in the digestive tract tend to peak during July and August (ProMED-mail 2003).

Young children are recognized as the primary reservoir of human enteroviruses (Moore 1982), with infants under one year of age accounting for $45 \%$ of all reported U.S. enteroviral isolates from 1997-1999 (CDC $2000 \mathrm{~b}$ ). Thus, youth is considered a predisposing factor for viral meningitis, with several studies showing age-specific incidence to be greater among young infants and school-aged children 5-10 years old (Rotbart 2000). The annual incidence of viral meningitis in children under one year-old in a large Finnish cohort was 219/100,000 children (Rotbart 2000). In our study, children $<5$ years old constituted $21 \%$ of all cases, and $23 \%$ of all cases were children aged 5-14 years.

Viral meningitis incidence among Nonwhites in the United States has been previously shown to be higher than that for Whites, a finding attributed to socioeconomic inequality and other confounding variables (Morens and Pallansch 1995). We too found elevated relative risk for viral meningitis among Blacks in Michigan. In other studies, infection rates have been reported to be higher in areas of crowding and among people of lower SES (Rotbart 2000), consistent with our finding of a significant correlation between cumulative incidence and population density. There was high viral meningitis incidence in Detroit, an area with higher population density, a large proportion of Blacks, and many residents of low SES. Being Black and of low SES are strongly autocorrelated in the Detroit area (Zenk 2003), and any separate effects they may have on incidence cannot be teased apart using our study design. These comparisons suggest that our case reports were consistent with those from other locations.

Our study departs from previous reports that found proportionately more enterovirus cases among males than females. Others have noted a male-tofemale sex ratio ranging from 1.3:1 (Rotbart 1995) to 2.5:1 (Morens and Pallansch 1995). In a viral meningitis outbreak in Romania, males were more often found to be ill $(\mathrm{p}=0.04)$ (CDC 2000a). However, in Michigan, males and females were diagnosed with viral meningitis in equal proportions.

Our finding that there may be a roughly triennial cycle of elevated incidence has some support in the literature. Interannual trends have been reported in poliovirus cases every three years in southern India (Morens and Pallansch 1995). Perhaps highly transmissible agents rapidly circulate in a population, quickly building high levels of population immunity. The observed pattern may also reflect the different periodicities of predominant enteroviral serotypes (CDC 2002). It is also possible that cyclic epidemics occur as the population reaches a threshold level of newly introduced susceptibles (e.g. very young children or immigrants), who reduce herd immunity and permit reappearance of intense transmission. This may be consistent with the large 2001 spatio-temporal cluster of viral meningitis cases (\#1, Fig. 5) that was dominated by children less than 10 years of age. Further studies of seasonal trends could improve understanding of factors that increase risk, hence providing an early warning of intense transmission periods. 
Regional environmental factors also may play a role in driving incidence cycles, as evidenced by the strong correlation in incidence between Michigan and Illinois. Strikas and colleagues (1986) grouped these two states together in their analysis of enterovirus isolates and identified a regional pattern of incidence. Other studies have reported geographical clustering of enteroviral strains in the U.S. and in Belgium (CDC 2003). However, any regional drivers are likely to be influenced by local factors, as the correlation between the annual incidences of Michigan and Indiana during the same time period was poor. Latitude, weather effects, and other environmental differences may be at play.

The most commonly identified virus isolated during the 2001 outbreak in Michigan was echovirus 13 (Table 1). This outbreak occurred in the context of increased detection of viral meningitis cases throughout the U.S. and across the globe. Michigan, along with Illinois, Tennessee, and Wisconsin, reported the most echovirus 13 isolates in 2001 in the country (CDC 2002), and epidemics of echovirus 13-associated viral meningitis took place in Louisiana, Mississippi, Montana, and Tennessee from April to July 2001, prompting outbreak investigations in these states (CDC 2001). Indeed, widespread circulation of echovirus 13 in the U.S. occurred for the first time in 2001 (CDC 2002, Noah and Reid 2002). Echovirus 13 was also very prevalent during 2000-2001 in Australia (ProMED-mail 2001, Quirk 2001) and Europe (Noah and Reid 2002).

Geography is represented using centroids when applying Kulldorff's Scan test, in this case the centroids of Michigan counties. The presence of the Great Lakes therefore prevented the scan statistic from assessing spatiotemporal clustering for the state as a whole. Furthermore, the geographic template employed in this test is a space-time cylinder of a given radius and duration. It is consequently capable of reporting clusters only as space-time cylinders, despite whatever the true shape of a particular cluster may be. The test could not accurately pinpoint, for instance, a cone-shaped spatiotemporal cluster, with an outbreak spreading out from an initial focus. If there is not enough power to detect a cluster of this shape, the spatial scan may continue to expand until it contains a large enough number of cases to declare a spurious cluster. This may explain why Cluster \#1 in this study encompassed such a large number of counties, and implies that, as always, caution is necessary when drawing statistical inferences. Kulldorff's Scan test also assumes that risk within the space-time cylinder is uniform despite the potential for variability in disease incidence rates within a cluster. It is therefore possible for the test to return false positives, as geographic areas with comparatively low incidence rates may be declared part of a cluster with high rates (Jacquez, GM [2005] Spatial Cluster Analysis. In: Wilson JP, Fotheringham S [eds] Handbook of Geographic Information Science, Blackwell Scientific, London [in review]). Other tests for space-time clustering could also be included in a future STIS for viral meningitis surveillance, to assess the sensitivity of identified clusters to the assumptions of the Kulldorff's Scan test.

Our study analyzed case data from a passive surveillance system and may not be a complete evaluation of all Michigan residents who had viral meningitis during the study period. It has been suggested that viral meningitis cases occur at more than ten times the number reported to the 
CDC (Rotbart 1995). We recognize that there could have been reporting variability among counties or regions due to unequal human and monetary resources or heightened concern and greater efforts at detection. For example, a ten-county district in the central region of Michigan (District \#10) was known to have intensified viral meningitis case finding during 2001 (personal communication via electronic mail, Dr. Patricia Somsel, August 2002). Another possible source of reporting bias might have involved the August 2001 detection of West Nile Virus (WNV) in birds in Michigan (Calisher 2001), which may have sensitized physicians to diagnosis and reporting of viral meningitis. WNV and enteroviruses have a similar seasonal pattern and both are associated with neurological signs and symptoms. However, WNV-associated meningitis tends to occur in adults (median age 46 years, CDC 2003), yet children are at highest risk for enteroviral meningitis. Furthermore, temporal analysis was based on date of report, not date of disease onset, and perhaps there was some variation among counties in submission of case records. Since the residence of each case was defined at the spatial scale of county, we were unable to track transmission or to draw conclusions about individual risk factors.

Despite these limitations, we have identified groups at elevated risk and larger spatio-temporal patterns that would be useful in hypothesis generation for future, prospective studies on epidemic patterns. However, our results suggest that improved knowledge of the causative agent may be essential to understanding transmission dynamics. To that end, more reliable and representative laboratory identification of specific pathogens is needed to determine whether outbreaks are caused by one or more viral strains. Indeed, the MDCH is embarking on an enterovirus laboratory surveillance program in reaction to recent outbreaks and this study's findings, in order to better characterize future enterovirus epidemiology. Clinical laboratories throughout the state are being encouraged to submit samples for typing on a weekly basis. Evidence suggests that numerous viruses and serotypes commonly cocirculate (CDC 2001) and that outbreaks of specific echovirus types are more widespread than previously believed (Noah and Reid 2002). Temporal trends in viral meningitis incidence stratified by virus type would have more interpretability than undifferentiated, amalgamated disease incidence.

The identification of spatial and temporal clusters in this study should encourage further research aimed at identifying local and socio-demographic influences on infectious disease agent transmission. We determined that counties with high incidence are consistently high (i.e. low coefficient of variation), suggesting intransient risk factors within these sites. There are no specific prevention or control measures known to reduce the transmission of non-polio enteroviruses beyond good hygienic practices (hand washing, disinfecting contaminated surfaces, and not sharing utensils or drinking containers, CDC 2003). Lacking specific options for preventing enterovirus epidemics, the incorporation of data and methods such as are described here into a STIS could prospectively focus surveillance activities and allocate limited resources for response to the districts with greatest need. Clinicians could thereby be better informed and equipped to assist health departments in responding to outbreaks by emphasizing preventive measures (Noah 1989). Such knowledge should guide further research and provide insights into prevention. STIS tools represent an exciting opportunity to enhance 
early warning analysis and public health action for viral meningitis and other diseases.

\section{References}

Calisher C (2001) West Nile Virus Surveillance 2001 - USA (18). ProMED-mail 20010825.2017. www.promedmail.org

CDC (1995) Summary of notifiable diseases, United States, 1994. Morbidity and Mortality Weekly Report, 43(53)

CDC (1997) Case definitions for infectious conditions under public health surveillance. $M M W R$ Recommendations and Reports, 46(RR-10):1-55

CDC (2000a) Outbreak of aseptic meningitis associated with multiple enterovirus serotypes Romania, 1999. Morbidity and Mortality Weekly Report, 49(29):669-671

CDC (2000b) Enterovirus surveillance - United States, 1997-1999. Morbidity and Mortality Weekly Report, 49(40):913-916

CDC (2001) Echovirus type 13-United States, 2001. Morbidity and Mortality Weekly Report, 50:777-780

CDC (2002) Enterovirus surveillance - United States, 2000-2001. Morbidity and Mortality Weekly Report, 51(46):1047-1049

CDC (2003) Outbreaks of aseptic meningitis associated with echoviruses 9 and 30 and preliminary surveillance reports on enterovirus activity - United States, 2003. Morbidity and Mortality Weekly Report, 52(32):761-764

Chin J (ed) (2000) Control of Communicable Diseases Manual. American Public Health Association, Washington, DC

Fosgate GT, Carpenter TE, Chomel BB, Case JT, DeBess EE, Reilly KF (2002) Time-space clustering of human brucellosis, California, 1973-1992. Emerging Infectious Diseases, 8(7):672-678

Glavanakov S, White DJ, Caraco T, Lapenis A, Robinson GR, Szymanski BK, Maniatty WA (2001) Lyme disease in New York State: Spatial pattern at a regional scale. American Journal of Tropical Medicine and Hygiene, 65(5):538-545

Huether S, McCance K (2000) Understanding Pathophysiology. Mosby, Inc, St. Louis

Kulldorff M (1997) A spatial scan statistic. Communications in Statistics - Theory and Methods, 26:1481-1496

Michigan Compiled Laws 333.5111 (1978). Public Acts, 368

Modlin JF (2004) Enteroviruses. In: Gershon AA, Hotez PJ, Katz SL (eds) Krugman's Infectious Diseases of Children, $11^{\text {th }}$ ed. Mosby, Philadelphia, PA

Moore M (1982) Enteroviral disease in the United States, 1970-1979. Journal of Infectious Diseases, 146(1):103-107

Morens D, Pallansch M (1995) Epidemiology. In: Rotbart HA (ed) Human Enterovirus Infections. ASM Press, Washington, DC

Noah N (1989) Cyclical patterns and predictability in infection. Epidemiology and Infection, 102:175-190

Noah N, Reid F (2002) Recent increases in incidence of echoviruses 13 and 30 around Europe. EuroSurveillance Weekly, 6(7)

ProMED-mail (2001) Meningitis, Echoviruses 13 \& 30 - Australia (Western). www.promedmail.org

ProMED-mail (2003) Meningitis, Viral - USA (Georgia). www.promedmail.org

Quirk M (2001) Echovirus to be considered in meningitis diagnosis. Lancet Infectious Diseases, $1: 220$

Rotbart HA (1995) Meningitis and encephalitis. In: Rotbart HA (ed) Human Enterovirus Infections. ASM Press, Washington, DC

Rotbart HA (2000) Viral meningitis. Seminars in Neurology, 20(3):277-292

Strikas RA, Anderson LJ, Parker RA (1986) Temporal and geographic patterns of isolates of nonpolio enterovirus in the United States, 1970-1983. Journal of Infectious Diseases, 153(2):346-351 
UniversalMAP (2000) Michigan County Atlas. Williamston, MI

U.S. Census, http://www.census.gov

Zenk SN (2003) Neighborhood racial composition and food access in metropolitan Detroit: Geographic information systems and spatial analysis. Ph.D. Dissertation in Health Behavior and Health Education Dept., School of Public Health, University of Michigan. Ann Arbor, MI 